O48 (continued)

Objective: The Dietary Guidelines (Guidelines) provides food-based guidance to help promote health and prevent chronic disease. Like nutrition science, dietary guidance continues to evolve.

Target Audience: The target audience is health professionals who implement the recommendations to reach consumers through programs, policies, and educational initiatives of Federal and other public health programs and services. The Guidelines has traditionally addressed Americans two years of age and older.

Description: By statute, every five years the U.S. Departments of Health and Human Services and of Agriculture jointly publish the Guidelines. While the core of the Guidelines has remained relatively consistent over time, it has evolved with the science from a focus on nutrients to foods and, more recently, to overall eating patterns. Additionally, the range of topics addressed has grown over time.

Rationale: The Guidelines evolves as a result of changes in the science, advancements in the process to review the science, and consideration of topics of public health importance current at the time. Future editions will continue to evolve. For example, beginning with the 9th edition, the Guidelines will address pregnancy and children from birth on.

Evaluation: Each edition of the Guidelines builds on the previous, with the scientific justifications for revisions informed by an in-depth review of the science.

Conclusions and Implications: The Guidelines serves as the evidence-based foundation and a critical tool for health professionals. Federal food programs and educational outreach rely on the Guidelines and its scientific underpinnings. Nutrition educators remain essential as communicators of the Guidelines through the sectors and settings where Americans make food choices.

Funding: US Department of Health and Human Services

\section{Effective Nutrition Interventions for Sustainable Maternal and Child Health: Lessons from the Countries that Achieved Their MDG 4 and 5 Targets}

Mary Murimi,PhD, LDN, RD, mary.murimi@ttu.edu, Texas Tech University, 1301 Akron Avenue, Lubbock, TX 79409; Ana Florencia Moyeda Carabaza, BS, Texas Tech University

Objective: The purpose of this study was to examine the factors that contributed to the attainment of Millennium Development Goal (MDG) 4 and 5, and determine the level of their intervention based on Maternal and Child Health $(\mathrm{MCH})$ conceptual framework.

Study Design, Setting, Participants: The study examined the interventions conducted by 15 countries, who achieved their MDG 4 target by at least 5.0\%, and MDG 5 target by $5.5 \%$, or both, and three countries that did not achieve their target for MDG 4 and 5 .

Results: The countries that succeeded in achieving their target for either MDG 4 or 5 intervened at three levels namely basic or societal level, underlying causes or community level, and direct causes levels. For example, Ethiopia, succeeded in the achievement of MDG 4 by improving basic services at the societal level, increasing healthcare services and providing specialized nutrition intervention to malnourished children. On the contrary, the countries that did not succeed did not address the basic causes, or did not implement their policies. Cameroon only worked at the direct causes, ignoring societal level and underlying causes. Botswana and Zimbabwe worked at the direct and underlying causes, and developed policies but failed to implement them.

Conclusions and Implications: It is critical that policymakers work on societal level problems, address the cause of the problems by addressing the underlying causes and focus on capacity building. Health professionals need to consider the motivations and limitations of the target population, including nutrition education. Targeting behavior change has the potential to result in sustainable $\mathrm{MCH}$.

Funding: None

\section{Reduction of Geophagic Practices and Nutrition Promotion in Women of Reproductive Age in Rural Kenya: A Pilot Study}

Aliza H. Stark,PhD, RD, Aliza.Stark@mail.huji.ac.il, The Hebrew University of Jerusalem, PO Box 12, Rehovot, IL Israel 76100; Sharon Iron-Segev, DSc, RD, The Hebrew University of Jerusalem; Janerose Nasimiyu Lusweti, MS, RD; Elizabeth Kamau-Mbuthia, PhD, Egerton University

Objectives: Geophagia, the deliberate consumption of earth or stones is widespread in sub-Saharan Africa. This practice is associated with exposure to heavy metals and parasites, micro-nutrient deficiencies and poor pregnancy outcomes. Our study aimed to characterize and reduce the practice of geophagia in Nakuru, Kenya and implement a nutrition education program to improve dietary intake.

Study Design, Setting, Participants: A prospective study was carried out in 135 women of reproductive age (15-49 y) known to practice geophagia. Baseline data was collected and evaluated knowledge, attitudes and practices (24-hour recall) regarding diet and geophagia. This was followed by a half-day educational intervention primarily based on the health-belief model.

Outcome Measures and Analysis: Descriptive statistics were used along with analyses of pre and post data using the Wilcoxon signed-rank test.

Results: Geophagia cut across all ages with 91(67.4\%) consuming $\geq 100 \mathrm{~g} /$ day. Strong cravings were reported by $81.5 \%$ as the primary reason for geophagic practices. The most common negative effect reported by participants was constipation (16.3\%). Nutrition education focusing on the dangers of geophagia elicited a decrease in amount of geophagic material consumed per day in $82(77 \%)$ of participants $(\mathrm{Z}=-7.914, \mathrm{p}<.001)$. Following the intervention, the proportion of participants who

Continued on page S21 